\title{
ANALYSIS OF A YIELD MANAGEMENT MODEL FOR ON DEMAND COMPUTING CENTERS
}

\author{
Yezekael Hayel ${ }^{1}$, Laura Wynter ${ }^{2}$ and Parijat Dube ${ }^{2}$ \\ ${ }^{1}$ INRIA/IRISA, ${ }^{2}$ IBM T.J. Watson Research Center
}

\begin{abstract}
The concept of yield management for IT infrastructures, and in particular for on demand IT utilities was recently introduced in [17]. The present paper provides a detailed analysis of that model, both in simplified cases where an analytical analysis is possible, and numerically on larger problem instances, and confirms the significant revenue benefit that can accrue through use of yield management in an IT on demand operating environment.
\end{abstract}

Key words: Yield management, optimization, discrete choice model

\section{INTRODUCTION}

In [17], a model for performing yield management in the context of IT provisioning was presented. This model is especially valuable in the context of an on demand operating environment. On demand IT services allow users to arrive at will into an IT system, in which scheduled jobs have already reserved the resource. Many potential applications of this type of IT infrastructure exist and a few are already in operation.

One example of an on demand IT service that exists today is the case of dynamic off-loading of web content. When a customer, such as an online retailer, experiences very heavy web site traffic, that retailer may have its excess traffic automatically redirected to an off-loading service. The process is invisible to end-users of the retailer. The yield management system as described in [17] could be used by the off-loading service provider to allocate its own capacity optimally and profitably. Many other potential applications of this technology are on the horizon: application service providers may run 
software applications on their own cluster of servers and allow customers, for a fee, to use those applications remotely. Yield management in this case sets capacity allocations (server use, storage, and bandwidth) and multiple price points to offer to those customers, depending on the available resource level of the service provider, as well as the market demand. Similarly, computing centers, that rent processing capacity to customers, can operate more profitably and more efficiently through use of a system of yield management.

Yield management is the technique used by the airline reservation systems to set booking limits on seats at each price class. In an on demand operating environment, customers and jobs, or service requests, arrive at random. Whereas some of the IT system resources are pre-reserved, the realtime arrival of new customers introduces the possibility to accomplish any number of desired service objectives. The service objectives are achieved by the yield management modules by setting prices judiciously, as a function of the resource utilization and user demand levels.

For example, when spare capacity exists, introducing dramatically low prices serves to introduce new demand into the system. Yield management allows the provider to set the dramatically low prices without sacrificing profits. On the contrary, it was proved in [17] that, under certain conditions, as the number of price points increases, the revenue increases. When usage costs are increasing linearly or sublinearly in the number of users, as is generally the case, profits can be shown to increase monotonically as the number of price points increases as well, in spite of the fact that some price points can be set below cost. The key to the remarkable increase in revenue and profits is that the number of slots available at each price point is limited, and set optimally so as to maximize revenue, given the demand model and available resource level.

In this paper, we analyze the model introduced in [17], both analytically and numerically. The analytical study is carried out on a simplified version of the model with only price points and fixed job sojourn times; as such it provides a bound on what can be said about the full-scale model. The numerical study then illustrates the benefits that the approach and our model can provide and confirms the tractability of the yield management paradigm to the IT On Demand context.

While the literature on airline yield management is clearly of great relevance to our problem of yield management in on demand IT services, there are notable differences which lead to significantly high complexity in our setting. First and foremost, the service under consideration in IT on demand does not have a fixed duration nor does it occupy a predetermined percentage of the resource capacity. That is, an airline seat is occupied precisely for 
the duration of the flight, and the number of seats to sell on any flight is known in advance.

On the other hand, in On Demand IT utilities, the duration of a job depends upon the type of server upon which it is run, and the number of servers, if it is parallelizable; further, the number of servers it requires depends upon the type of servers that are used. In other words both the capacity needed and the time taken by a job are not simple, exogenous parameters in the compute On Demand yield management problem. Some features of this time variability can be observed in other sectors, such as hotellerie, restaurant yield management, and even golf course yield management (see, for example, [7] and [8], and other references by those authors). Nonetheless, the capacity and percentage of capacity occupied in these latter examples are still fixed and exogenous, as opposed to the setting with which we are faced.

Research work on the pricing of information and telecommunication services, such as the pricing strategies of internet service providers (ISPs) has traditionally considered some of these issues of job duration and capacity occupation through queuing formulae. The literature on that and related areas is quite vast and a thorough survey of it is not the focus of our work here, but a few relevant references are $[4,5,6,11,12,13,15]$. The difference between the decisions optimized in those and related work is the degree of segmentation. In the Internet pricing world, a single price per type of service is proposed. It is sometimes the case that multiple qualities of service (QoS) are discussed, but in that case, each QoS level has associated with it a single, fixed price. The number of such price levels is generally limited to three, for example, gold, silver, and bronze-level service. The yield management strategy takes customer segmentation to a much finer level, and does so through the incorporation of demand models.

In short, what makes this yield management approach to IT resource pricing and management so appealing is that it allows an IT provider to very tightly couple her IT resources to the demand. By so doing, the IT provider can substantially increase profit margins on the existing capacity, and furthermore modify the demand through targeted pricing/offering definition. As a by-product, the IT provider can accomplish other objectives, such as smoothing the demand over time, to avoid, for example, weekly peaks and excess spare capacity on the week-ends. Existing approaches to pricing IT resources that do not make use of this yield management approach do not have sufficient segmentation, in time or in the description of user demand, to accomplish these management objectives.

The structure of the paper is as follows. In Section 2, we propose and study an analytically tractable version of the model of [17] so as to gain qualitative insights on the nature of the problem. Section 3 contains a numerical study of the model. Finally, we conclude in Section 4. 


\section{THE MODEL}

In this paper, we shall assume that the on demand service infrastructure is composed of a pool of homogeneous nodes (processing units) to allocate to different fee classes. The optimization problem that we will need to solve is then the following: in a particular time epoch (in this paper we consider only one), we would like to reserve the available resource for the different fee classes. The resource should be allocated so as to maximize expected provider profits, that is, expected revenue less expected costs, where expected provider revenue is related to the distributions of different customer arrival types, their preferences (in terms of service/price trade-offs) as well as their service requirements, and to the number of nodes assigned to each fee class, on each server type.

Fee classes are defined as follows: for an identical resource several different prices may coexist; each fee class then has a maximal number of users, and once that number is reached within the time period for that fee class, new requests are offered only the next higher level fee for that resource.

Resources are also defined in a broad way. While a server and storage are clearly aspects of the resource, the service-level (SLA) parameters are as well, such as availability, advance notice, penalties in case of nonsatisfaction of service level by the provider, etc. The broad scope of the resource in this manner allows the price differentiation to become still finergrained; that is, for an identical server/storage combination, different SL offerings create new sets of fee classes.

With respect to notation, $T_{c}$ is the (here, deterministic) sojourn time of a job of class $c=1 \ldots C$ in the system. The decision variables, denoted by $n_{k}$, represent the number of resource slots to reserve for price segment $k=1$... $K$. Further, let $n=\left(n_{k}\right)$ be the vector of all $n_{k} s$, and $N$ the total amount of resource; while this could be extended to heterogeneous resource types, here for notational simplicity, we make use of a single resource type, of which there are $N$ units. In general, job sojourn time depends on the workload, or size, of the job, $W$, the number of slots allocated $\boldsymbol{n}_{\boldsymbol{k}}$, and the type of job, $c$. However, modeling explicitly that dependence leads to a non-convex feasible set, in that the sojourn time appears in the definition of the constraints. Therefore, in this paper, we have assumed the job sojourn time, $T_{c}$, to be externally provided.

The choice probability of a user with job class $c$ accepting a slot of segment-type $k$ is given in general by $P_{k}^{c}(W, n)$, but again for simplicity, we have suppressed dependency on the particular workload, and made use of a choice probability of the form $P_{k}^{c}(n)$. The probability of an arrival of job class $c$ is given by $\mathrm{G}_{c}$. Recall that we are considering here only one time epoch. The parameters $r_{k}$ are the price points of the resource. By enumerating a 
wide range of such prices, the optimization model works by identifying those price segments which are most profitable to offer, given the characteristics of the available demand and resource levels.

As stated earlier, we consider, in this paper, a simplified model in which two different prices per node are offered, i.e. $\boldsymbol{r}_{l} \neq \boldsymbol{r}_{2}$. Furthermore, we shall consider two different user, or job, classes, $c=1,2$. Under these simplifications, we shall be able to examine the model analytically and obtain bounds on the decision variables. The simplified yield management for IT resource model can be expressed as:

$$
\begin{aligned}
\max _{n_{1}, n_{2} \geq 0} F\left(n_{1}, n_{2}\right) & =\sum_{c=1}^{C} \sum_{k=1}^{2} T_{c} r_{k} n_{k} P_{k}^{c}(n) \Gamma_{c} . \\
n_{1}+n_{2} & \leq N
\end{aligned}
$$

Alternatively, one can assume that the resource limits are soft constraints and include the possibility to surpass those limits, at a cost associated with having to make use of remote resources or to pay a penalty to the customers.

While these results cannot be extended in general to any number of parameters, they, along with the larger-scale numerical results, provide valuable insight into the nature of the problem under study.

To model the behavior of customers, or job requests, we introduce a deterministic discrete choice function That is, we use the normalized ratio of the utility of choice $i$ to the sum of all choice utilities, that is, $\forall c$, and $\forall k$,

$$
P_{k}^{c}(n)=\frac{1}{K-1}\left(1-\frac{U_{k}^{c}\left(n_{k}\right)}{\sum_{j=1}^{K} U_{j}^{c}\left(n_{j}\right)}\right)
$$

The first term normalizes the quantities $P_{c}^{k}(n)$ so that they sum to 1 for each customer class, $c$. The second term is expressed as 1 - ratio, since the $U_{c}^{k}(n)$ are actually dis-utilities and hence decreasing in price and delay. With only two price segments, we have, for $c=1,2$, 


$$
P_{1}^{c}(n)=\frac{U_{2}^{c}\left(n_{2}\right)}{U_{1}^{c}\left(n_{1}\right)+U_{2}^{c}\left(n_{2}\right)} \text { and } P_{2}^{c}=\frac{U_{1}^{c}\left(n_{1}\right)}{U_{1}^{c}\left(n_{1}\right)+U_{2}^{c}\left(n_{2}\right)}
$$

where the (dis-)utility functions are:

$$
U_{k}^{c}\left(U_{1} \quad U_{k}^{c}\left(n_{k}\right)=\zeta_{1} T_{c} r_{k} n_{k}+\zeta_{2} T_{c} .\right.
$$

The parameters $\zeta_{1}$ and $\zeta_{2}$ are constants that define the price-time tradeoffs, and render the utility $U$ unitless. There are different ways to define these parameters, but we have chosen to use a single, deterministic, parameter vector for all customers. Recall also that $T_{c}$ is a constant that depends only on job class. The utility function is thus linear in the decision variable, $n_{k}$.

Explicitly including the deterministic discrete choice model into the objective function for this two-price-segment model, we obtain:

$$
\begin{aligned}
\max _{n_{1}, n_{2} \geq 0} F\left(n_{1}, n_{2}\right) & =\sum_{c=1}^{C} \Gamma_{c} T_{c}\left(f\left(n_{1}, n_{2}\right)+g\left(n_{1}, n_{2}\right)\right) \\
n_{1}+n_{2} & \leq N
\end{aligned}
$$

with

$$
f\left(n_{1}, n_{2}\right)=r_{1} \frac{\zeta_{1} r_{2} n_{1} n_{2}+\zeta_{2} n_{1}}{\zeta_{1} r_{1} n_{1}+\zeta_{1} r_{2} n_{2}+2 \zeta_{2}}
$$

and

$$
g\left(n_{1}, n_{2}\right)=r_{2} \frac{\zeta_{1} r_{1} n_{1} n_{2}+\zeta_{2} n_{2}}{\zeta_{1} r_{1} n_{1}+\zeta_{1} r_{2} n_{2}+2 \zeta_{2}}
$$

As the revenue increases in the number of nodes reserved to all price segments, the inequality constraint expressed in (1.2) will be active. In this simplified setting, we have the following result.

Proposition 1 The nonlinear yield management reservation problem of

\section{1 has a unique maximum}

Proof: We shall seek a maximum of the objective function on the induced, linear subspace defined by the active capacity constraints. To do so, we shall define the Hessian of the objective function augmented by the Lagrange multipliers and capacity constraints; this Hessian matrix is sometimes referred to as the "bordered Hessian". 
First, the Lagrangian function is :

$$
L\left(n_{1}, n_{2}, \mu\right)=\sum_{c=1}^{C} \Gamma_{c} T_{c} \cdot\left(f\left(n_{1}, n_{2}\right)+g\left(n_{1}, n_{2}\right)\right)-\mu G\left(n_{1}, n_{2}\right),
$$

with Lagrange multiplier $\mu \in \mathfrak{R}_{+}$, and constraint function: $G\left(n_{l}, n_{2}\right)=$ $n_{1}+n_{2}-N$.

Again, as mentioned, at optimality, the capacity constraint, $G\left(n_{1}, n_{2}\right) \leq 0$, will be active, hence $n_{1}+n_{2}=N$. After some manipulation of the Lagrangian, we obtain the following system:

$$
\left\{\begin{array}{c}
\frac{r_{1} \sum_{c=1}^{O} \Gamma_{c} T_{0}\left(2 \zeta_{1}^{2} r_{2}^{2} n_{2}^{2}+4 \zeta_{1} \zeta_{2} r_{2} n_{2}+2 \zeta_{2}^{2}\right)}{\left(\zeta_{1} r_{1} n_{1}+\zeta_{1} r_{2} n_{2}+2 \zeta_{2}\right)^{2}}-\mu=0, \\
\frac{r_{2} \sum_{c=1}^{O} \Gamma_{c} T_{0}\left(2 \zeta_{1}^{2} r_{1}^{2} n_{1}^{3}+4 \zeta_{1} \zeta_{2} r_{1} n_{1}+2 \zeta_{2}^{2}\right)}{\left(\zeta_{1} r_{1} n_{1}+\zeta_{1} r_{2} n_{2}+2 \zeta_{2}\right)^{2}}-\mu=0, \\
n_{1}+n_{2}=N,
\end{array}\right.
$$

from which we obtain:

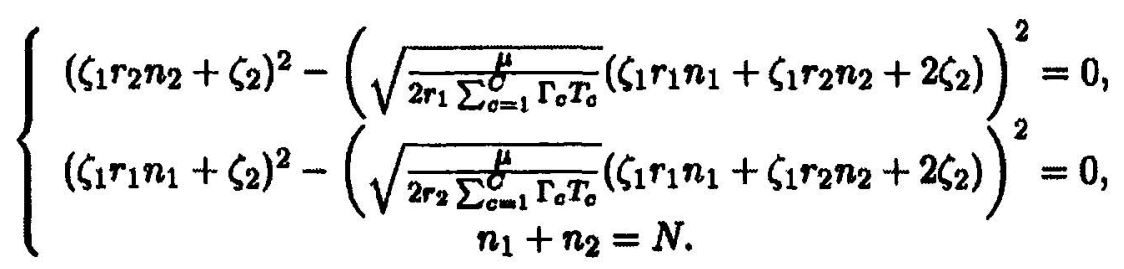

Noting that each of the first two equations is a quadratic, we obtain four sets of equations. However, of those, only one has a feasible solution, which we shall show is the unique maximum. The solution is given by:

$$
\left(n_{1}^{*}, n_{2}^{*}\right)=\left(N \frac{\sqrt{r_{2}}}{\sqrt{r_{1}}+\sqrt{r_{2}}}+\mathcal{H}, N \frac{\sqrt{r_{1}}}{\sqrt{r_{1}}+\sqrt{r_{2}}}-\mathcal{H}\right)
$$

with $\mathcal{H}=\frac{\zeta_{2}}{\zeta_{1} r_{2}} \frac{\sqrt{r_{2}}}{\sqrt{r_{1}}+\sqrt{r_{2}}}\left(1-\sqrt{\frac{r_{2}}{r_{1}}}\right)$. The optimal Lagrangian multiplier, $\mu^{*}$, is then:

$$
\mu^{*}=2 \sum_{c=1}^{C} \Gamma_{c} T_{c} \frac{r_{1} r_{2}}{\left(\sqrt{r_{1}}+\sqrt{r_{2}}\right)^{2}} .
$$

Additionally, as $\boldsymbol{n}_{1}^{*}$ and $\boldsymbol{n}_{2}^{*}$ are non-negative, we obtain two necessary conditions on the feasible range of prices, $\boldsymbol{r}_{1}$ and $\boldsymbol{r}_{2}$, for a solution: 


$$
\begin{aligned}
& r_{1} \geq \frac{r_{2}}{\left(1+N \frac{\zeta_{1} r_{2}}{\zeta_{2}}\right)^{2}}, \\
& r_{1} \leq r_{2}\left(1+N \frac{\zeta_{1} r_{2}}{\zeta_{2}}\right)^{2} .
\end{aligned}
$$

Thus, depending on the values of the problem constants, we can determine the range of prices for which a solution exists. Figure 1 illustrates this range for a capacity level $N=10$, and for a range of a new parameter, $\zeta$, which is defined as $\zeta=\zeta_{1} / \zeta_{2}$

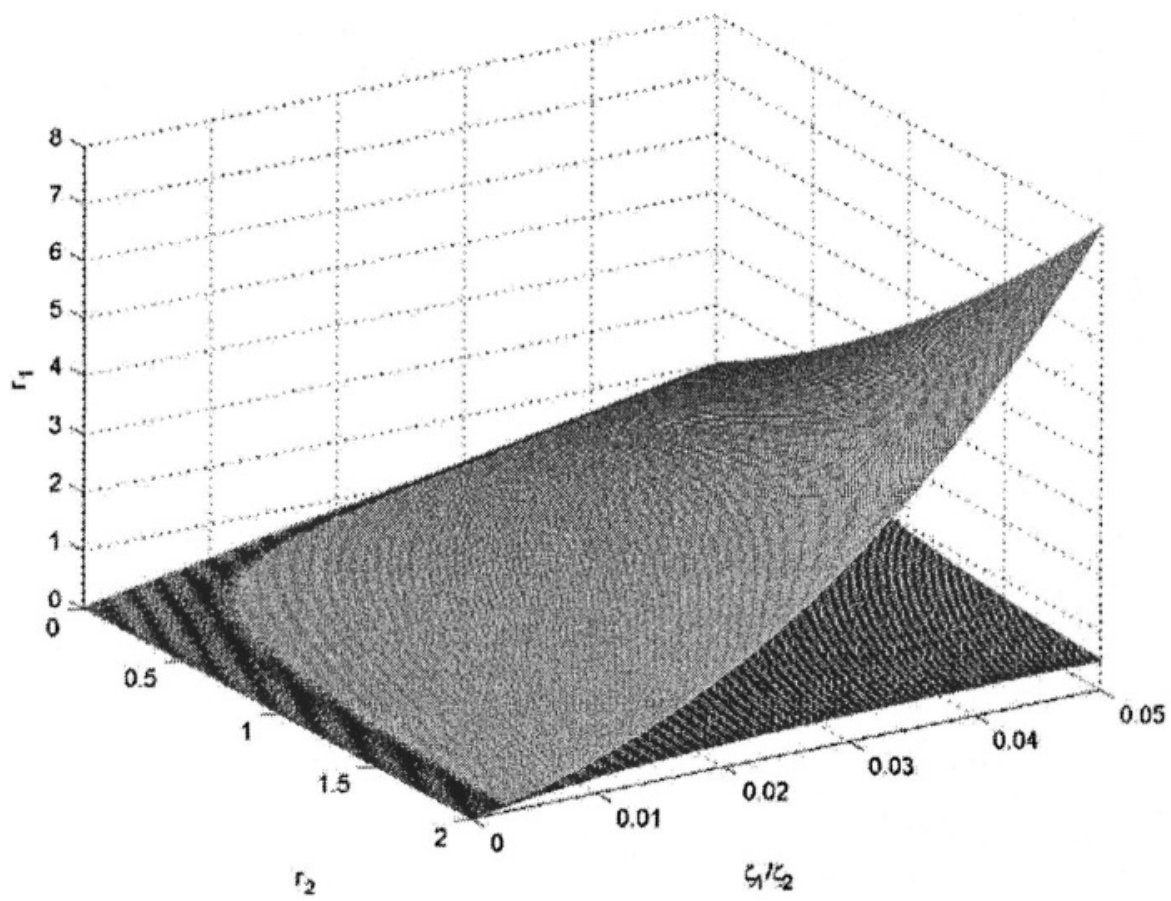

Figure 1. Existence area of the solution when $\mathrm{N}=10$

To prove that the solution we have found is indeed a maximum, we examine the second-order conditions for this constrained maximization problem. The Hessian of the Lagrange function is defined by: 


$$
\bar{D}(z)=\left(\begin{array}{ccc}
0 & G_{n_{1}} & G_{n_{2}} \\
G_{n_{1}} & L_{n_{1} n_{1}} & L_{n_{1} n_{2}} \\
G_{n_{2}} & L_{n_{2} n_{1}} & L_{n_{2} n_{2}}
\end{array}\right),
$$

where $\boldsymbol{z}:=\left[\boldsymbol{\mu}, \boldsymbol{n}_{1}, \boldsymbol{n}_{2}\right]$ We then must determine whether the Hessian of the Lagrangian function $\bar{D}$, evaluated at the possible optimum, is negative semidefinite subject to the linear constraint $w^{\prime} D w \leq 0$ for all $w \in \mathfrak{R}^{3}$ satisfying $\left(\partial G / \partial_{i}\right)\left(w_{i}\right)=0$, where the index on $z$ indicates the element of that vector, and similarly for $w$. This negative-definiteness condition implies that the principle minor determinants of the Hessian of the Lagrange function alternate in sign. In our two-variable, one constraint, setting, the second-order condition amounts to the $3 \times 3$ determinant (including the constraint) being positive. Note, from the alternating signs, that in a three-variable, oneconstraint problem, the corresponding $4 \times 4$ determinant would need to be negative, etc...

The second derivatives of the Lagrangian function are:

$$
\begin{aligned}
& L_{n_{1} n_{1}}=\frac{\theta^{2} L}{a_{2}}\left(n_{1}, n_{2}\right)=-S^{C} \Gamma_{\mathrm{c}} T_{\mathrm{v}}\left(2 \zeta_{1}^{2} r_{1} r_{2}^{2} n_{2}^{2}+4 \zeta_{1} \zeta_{2} r_{1} r_{2} n_{2}+2 r_{1} \zeta_{2}^{\prime}\right) \frac{2 \zeta_{1} r_{1}}{\left.{ }_{1}{ }_{1} r_{1} n_{1}+\zeta_{1} r_{2} n_{2}+2 \zeta_{2}\right)^{8}}, \\
& |\bar{D}|=-G_{n_{1}}^{2} L_{n_{1} n_{1}}+2 G_{n_{1}} G_{n_{2}} L_{n_{1} n_{2}}-G_{n_{2}}^{2} L_{n_{2} n_{2}} \text {, }
\end{aligned}
$$

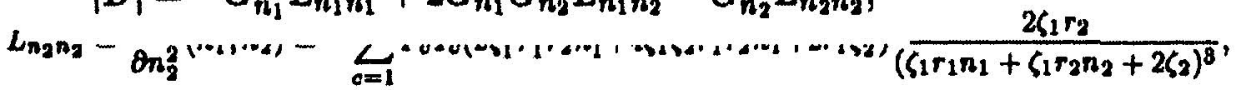

$$
\begin{aligned}
& L_{n_{1} n_{2}}=\frac{\partial^{2} L}{\partial n_{1} n_{2}}\left(n_{1}, n_{2}\right)=\frac{\sum_{c=1}^{C} \Gamma_{c} T_{c}}{\left(\zeta_{1} r_{1} n_{1}+\zeta_{1} r_{2} n_{2}+2 \zeta_{2}\right)^{3}}\left[4 \zeta_{1}^{4} r_{1}^{8} r_{2}^{2} n_{1}^{2} n_{2}+\right. \\
& +4 \zeta_{1}^{4} r_{1}^{2} r_{2}^{8} n_{1} n_{2}^{2}+16 \zeta_{1}^{\mathrm{g}} C_{2} r_{1}^{2} r_{2}^{2} n_{1} n_{2}+8 \zeta_{1}^{\mathrm{S}} C_{2} r_{1} r_{2}^{8} n_{2}^{2}+ \\
& \left.+4 \zeta^{8} \zeta_{2} r_{1}^{8} r_{2} n_{1}^{2}+8 \zeta_{1} \zeta_{2}^{8} r_{1} r_{2}+12 \zeta_{1}^{2} \zeta_{2}^{2} r_{1}^{2} r_{2} n_{1}+12 \zeta_{1}^{2} \zeta_{2}^{2} r_{1} r_{2}^{2} n_{2}\right]
\end{aligned}
$$

The determinant of the Hessian is:

where $G_{n l}=G_{n 2}=1$. Thus we obtain that the determinant of $D$ is indeed positive for all $n_{l} \geq 0$ and $n_{2} \geq 0$, and we conclude that $\left(\boldsymbol{n}_{1}^{*}, \boldsymbol{n}_{2}^{*}\right)$ is indeed a maximum over the frontier.

\section{YIELD MANAGEMENT FOR WEB TRANSAC- TION DATA}

We apply our optimization model to web transaction data over an eightday horizon. The data we have does not include job durations; therefore we consider all jobs to have unit duration (here, the time unit is one hour). The yield management reservation (YMR) system functions similarly when jobs have heterogeneous durations. The subscription works as follows: some us- 
ers, not willing to pay high prices for service, subscribe only if they can obtain the service at an acceptable price level to them. If no such acceptable price is available (not offered, or the maximal quantity is attained) then those customers" go elsewhere". Other users with higher willingness-to-pay can still subscribe, until their threshold is reached, and so on. Therefore, depending upon the prices offered, and the available quantities of each, a different share of the market can be captured, and revenue will thus vary as well.

The objective of the YMR system is to determine which offerings to propose to customers, and the optimal quantity to propose of each offering, so as to maximize potential revenue. Here, we illustrate the output of the YMR system in terms of the optimal number of slots to propose at each of the price levels, and then compare the resulting revenue stream with the basecase, in which a single price per QoS is charged.

The transaction data represents the demand at each point of time. The YMR model allows for the possibility that a user does not accept any of the offerings proposed. In this series of examples, we have considered a single QoS level and multiple prices for that QoS, with the quantities of slots available at each price limited, by a number to be determined by the YMR. Possible price levels are determined in advance, with not necessarily all price levels open in the optimal solution; the possible set of price points are given in Table 3. On the left column of the table, we consider a variable number of price segments in each optimization run, from 1 single price (be it high, medium, or low) to 6 price points.

Table 1. Input data on the possible prices for each simulation, in which 1 to 6 price segments are offered to customers, in limited quantities

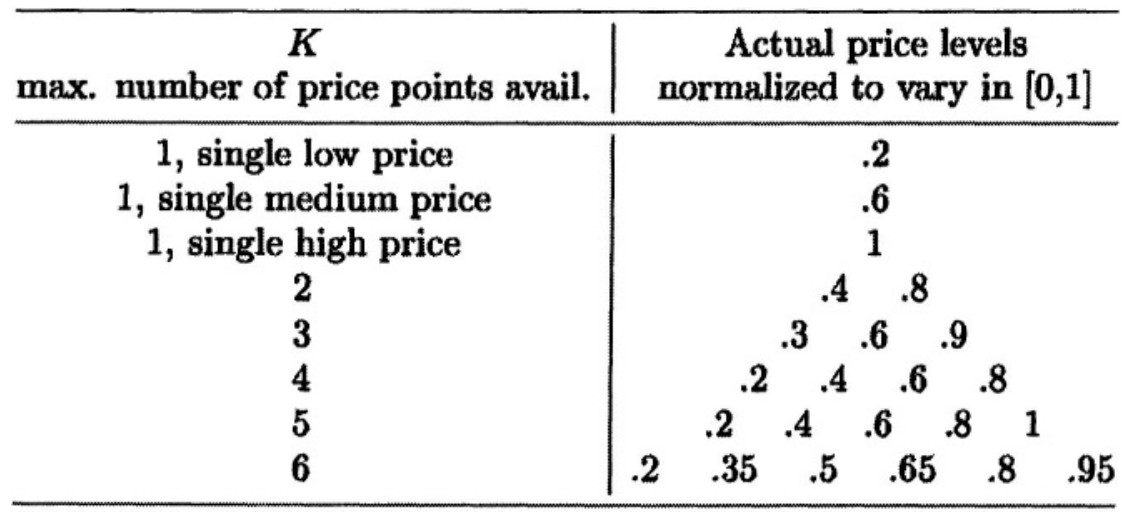

The first figure illustrates the optimal revenue over time when 2 to 6 price segments are made available to customers, in limited quantities. Note that the topmost curve is the total demand, not the revenue. The revenue 
accrued under each simulation ( 2 through 6 price segments on offer) is indicated in the lower series of curves. The larger numbers of price segments (5-6) clearly gives higher revenue during peak periods, whereas during periods of lower use, 2-3 price segments on offer is optimal.

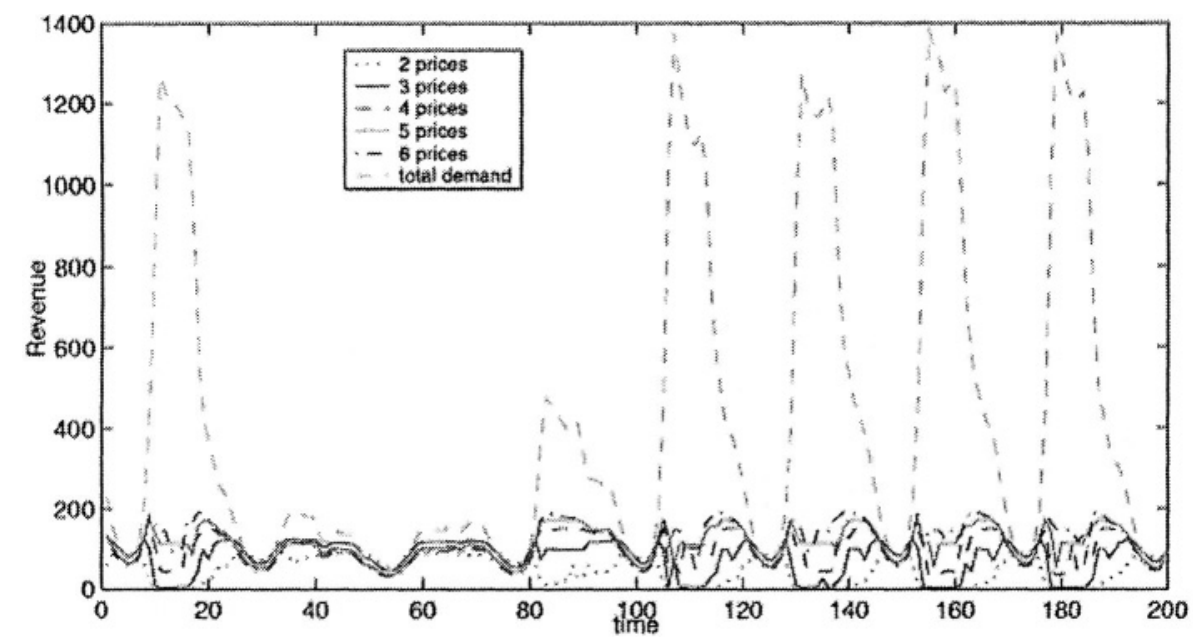

Figure 2. Revenue stream for different numbers of price segments on offer.

The highest curve gives the total demand over time, and serves only to illustrate the peaks and valleys.

Figure 3 summarizes the data of the first two figures for certain time periods, for increased clarity. In particular, we have chosen 5 time periods, with alternating peak flows and off-peak flows, to illustrate how the optimal number of price segments to offer varies.

The last set of figures (Figures 4 and 5) compares the revenue when only one price segment is offered (for three cases: a low, medium or high price) with a strategy of offering five price points (irrespective of the demand level).

Observe that the 5-price-segment offering is always superior to offering a single price, irrespective of whether a low, medium, or high single price is offered. Furthermore, from the above figures, we know that the YMR system would not suggest always proposing 5 price segments irrespective of the load level, but would allow further revenue increase by modulating the number of segments to offer with the demand level (less segments when demand is low, more when it is high). 
Figures 5 illustrates the optimal numbers of slots to offer at each of those 5 price levels and demonstrates that the optimal number of price segments varies with the total demand, or load, in that the higher

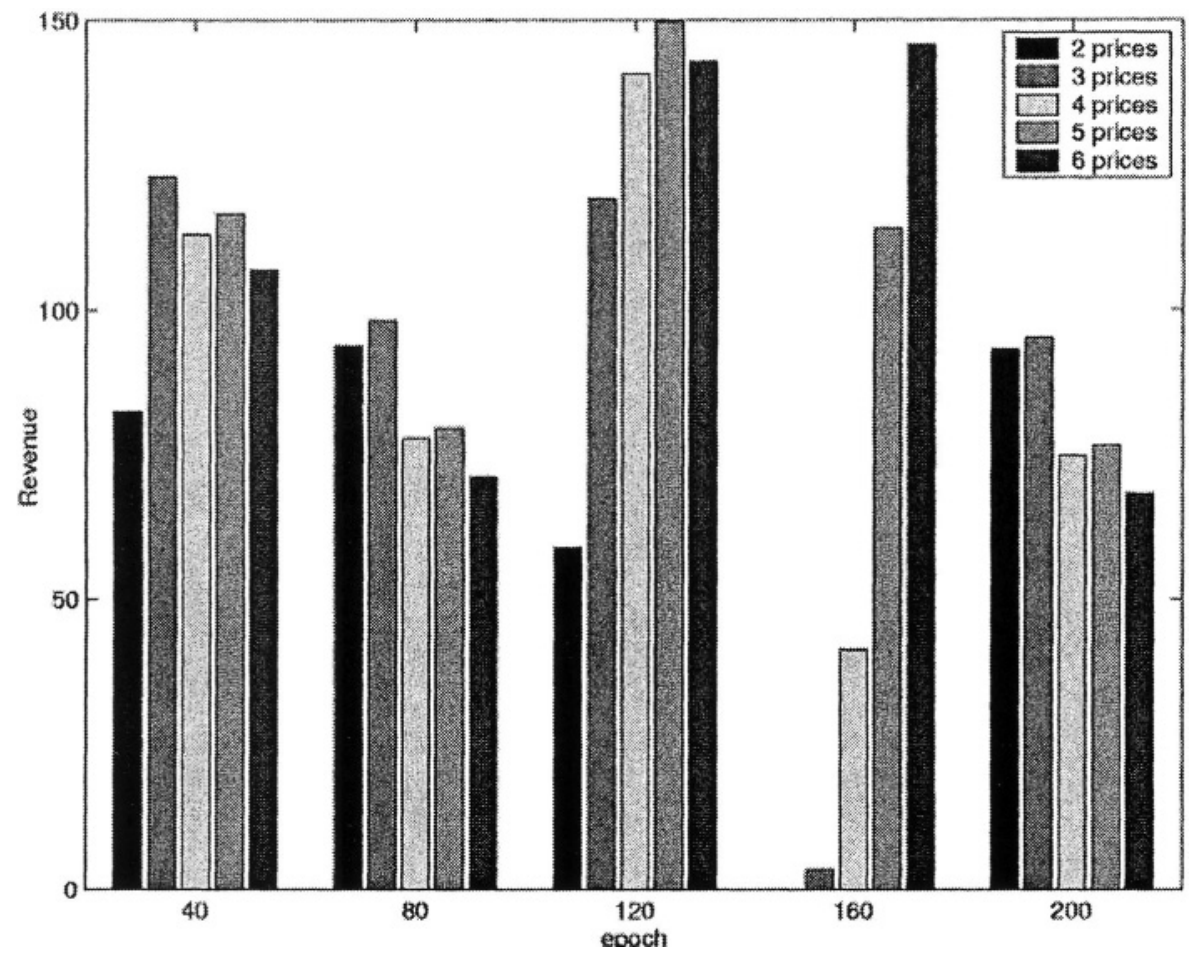

Figure 3. Optimal revenue for 5 diflferent time periods (periods oflf-peak(40), medium (80), peak (120), peak (160) and oflf-peak (200)) over the 5 diflferent YMR strategies (offering 2-6 price segments).

the demand, the higher the number of segments should be to maximize revenue. This implies furthermore that the YMR should be re-run as new and better demand data become available. Figure 5 shows the entire breakdown over the 8-day time horizon.

\section{CONCLUSION}

In this paper, we have presented a yield management model for on demand IT services such as e-commerce services, or data processing centers. We have provided an approach for determining an optimal reservation of resources in order to maximize expected revenue, as well as a detailed ana- 
lytical analysis of the resulting optimization problem when the number of class of prices is small. Finally, we provide numerical results on time series data of web transactions that illustrate the impact of the approach on service provider revenue.

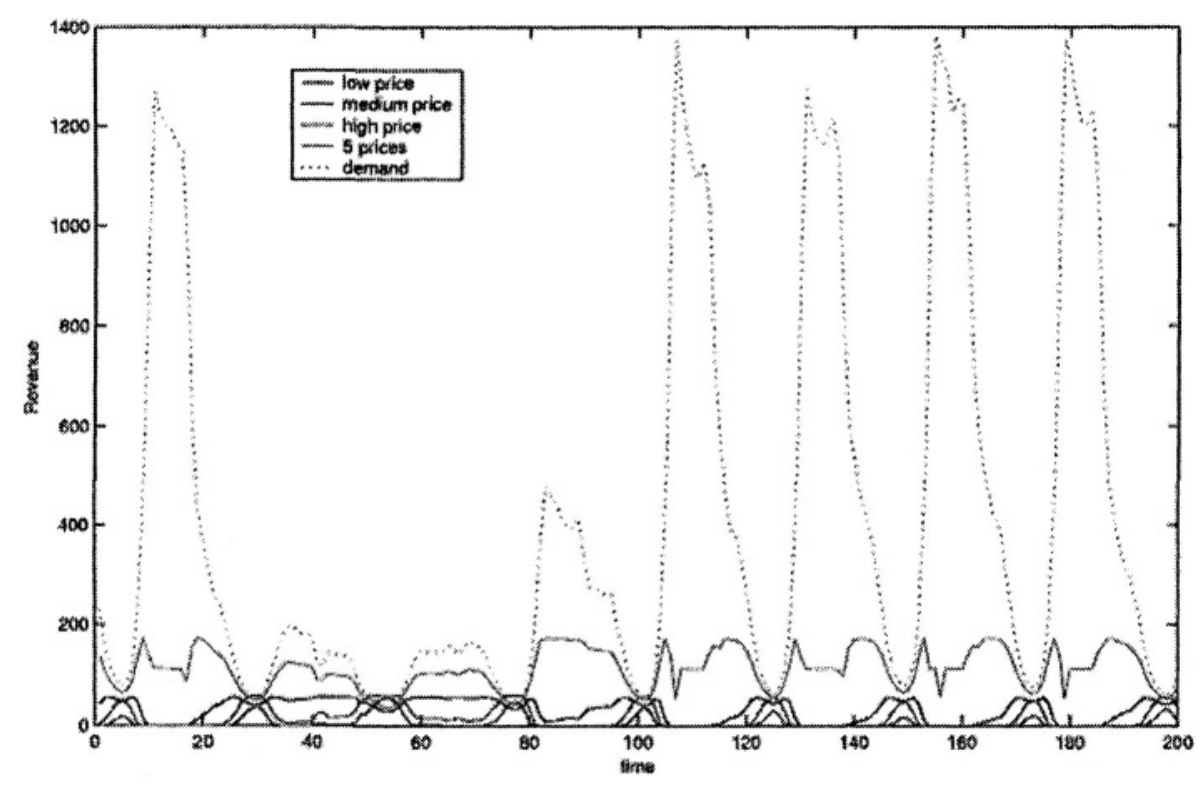

Figure 4. Comparison of YMR strategy of offering 5 price segments with a single-price offering, where the single price is either low, medium, or high

\section{REFERENCES}

[1] P.P. Belobaba, Airline yield management: an overview of seat inventory control. Transportation Science, 21, 63-73, 1987.

[2] M. Ben Akiva and S. Lerman Discrete choice Analysis: Theory and application to travel demand. MIT Press, Cambridge, 1985.

[3] P. Davis, Airline ties profitability yield to management, SIAM News, 27-5, 1994.

[4] R. El Azouzi, A. Altman, and L. Wynter, Telecommunications network equilibrium with price and quality-of-service charactersitics to appear in the Proceedings of the 18th International Teletraffic Conference (ITC), 2003.

[5] R. J. Gibbens and F. P. Kelly, Resource pricing and the evolution of congestion control, Automatica, 35(12), 1969-1985, 1999.

[6] A. Gupta, D. Stahl, and A. Whinston, The Economics of Network Management, Communications of the ACM, 42(5), 57-63, 1999. 
[7] S.E. Kimes, D.I. Barrash and J.E. Alexander, Developing a restaurant revenue management strategy, Cornell Hotel and Restaurant Administration Quarterly, 40-5, 18-29, 1999.

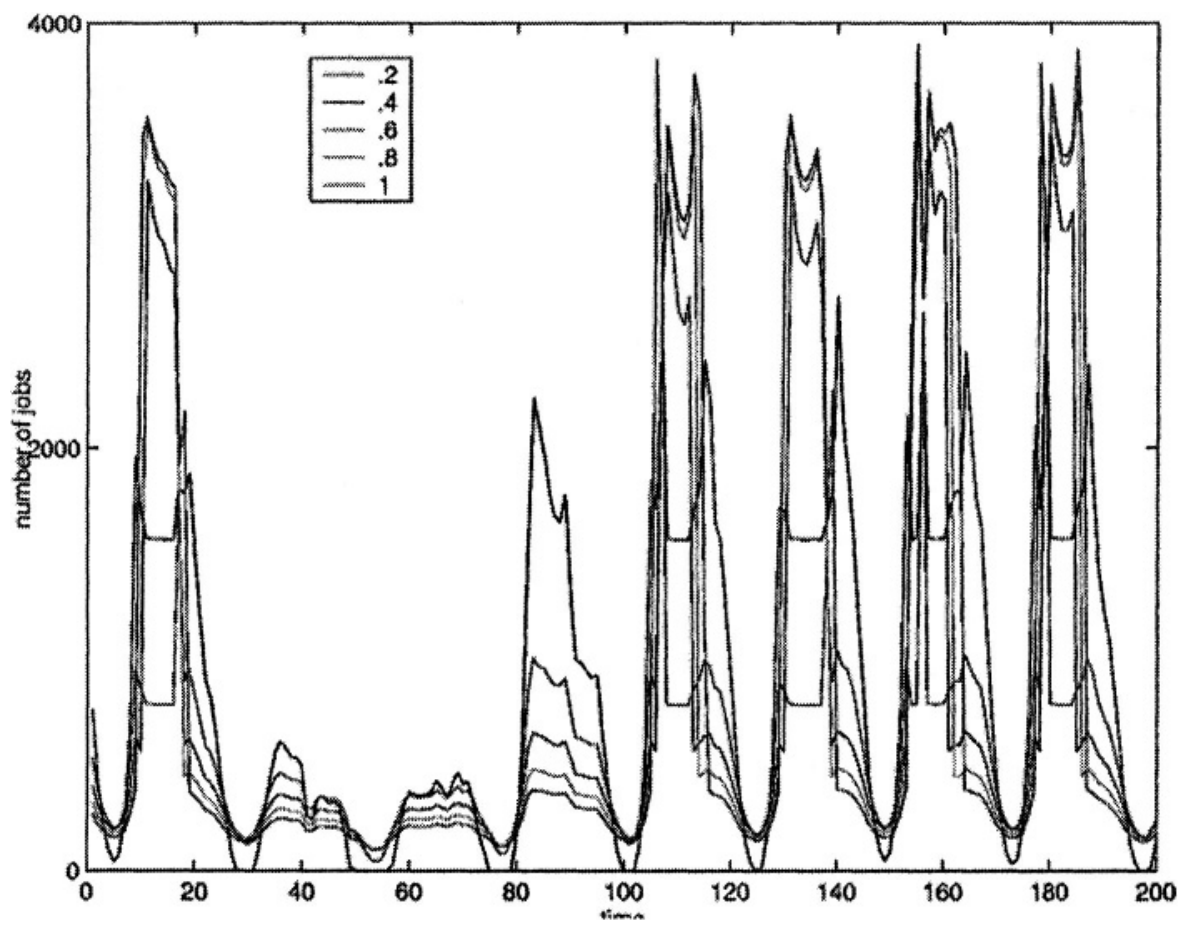

Figure 5. Number of slots to oflfer at each of the 5 prices segments over time.

[8] S.E. Kimes, Revenue management on the links: applying yield management to the golf course, Cornell Hotel and Restaurant Administration Quarterly, 41-1, 120-127, 2001.

[9] A.J. Kleywegt, An optimal control problem ofdynamic procing Georgia Tech Research Report, 2001.

[10] K. Littlewood, Forecasting and control of passengers 12th AGI-FORS Symposium Proceedings, 95-128, 1972.

[11] Z. Liu, L. Wynter, and C. Xia, Pricing information services in a competitive market: avoiding price wars INRIA Research Report 4679. Available at www.inria.fr/rrrt/rr4679.html. Also in proc. of 4th ACM conference on Electronic commerce, San Diego, CA, USA, June 2003

[12] J. Mackie-Mason and H. Varian Pricing the Internet in Public Access to the Internet, B. Kahn and J. Keller, (Eds.) Prentice Hall, Englewood Cliffs, 1995.

[13] A. Odlyzko, Paris metro pricing for the Internet in Proceedings of the ACM Conference on Electronic Commerce, 140-147, 1999. 